\title{
Editorial: Recent Advances in Mining Intelligence and Context-Awareness on loT-Based Platforms
}

\author{
Cheonshik Kim ${ }^{1}$ - Byung-Gyu Kim² - Joel J. P. C. Rodrigues ${ }^{3,4,5}$
}

Published online: 13 November 2018

(C) Springer Science+Business Media, LLC, part of Springer Nature 2018

To develop innovative and intelligent systems or programs suitable for the future, a theoretical approach is needed to recognize problems that are appropriate for various situations. To this end, many researchers are developing various types of systems based on their new ideas. Recently, Deep Learning has been applied to various fields by improving the performance of neural network technology which is a conventional artificial intelligence function. By sharing an open source (e.g., TensorFlow) for deep learning, we are able to utilize it for our own purposes without difficulty and obtain better research results than our previous research results. Deep Learning can work with sensors that can gather a lot of information. In addition, sensor networks have expanded over the past few years in a variety of areas such as heritage conservation, environmental movement and human activity. Therefore, integration of intelligent machine learning system and sensor data is a natural choice and sensor based recognition technology with artificial intelligence is emerging as an important research field.

This special issue aims to share the latest machine learning research results, including results from various

Cheonshik Kim

mipsan@paran.com

Byung-Gyu Kim

bg.kim@sookmyung.ac.kr

Joel J. P. C. Rodrigues

joeljr@ieee.org

1 Sejong University, 209, Eungdong-ro, Gwangjin-gu, Seoul, South Korea

2 Sookmyung Women's University, Cheongpa-ro 47-gil 100, Yongsan-gu, Seoul, South Korea

3 National Institute of Telecommunications (Inatel), Santa Rita do Sapucaí, Brazil

4 Instituto de Telecomunicações, Lisboa, Portugal

5 University of Fortaleza (UNIFOR), Fortaleza, Brazil sensor-based studies, with many researchers. This study has just initiated in both industry and academia, but with great fervor all over the world.

This special issue features six selected papers with high quality. The first article, "An Intelligent Product Recommendation Model to Reflect the Recent Purchasing Patterns of Customers" [1] proposed a new product recommendation model to reflect the recent purchasing patterns of customers. In addition, various data mining classifiers such as the decision tree, neural network, support vector machine, random forest, and rotation forest are applied, and a sliding-window scheme is considered to construct the recommendation model.

The second article titled "SMinder: Detect a Leftbehind Phone using Sensor-based Context Awareness" [2] presented a technique to detect the situation of forgetting to take the phone when getting off the car. SMinder reminds the user with high accuracy and minimum energy consumption, making it realistic for real-world use.

In the next article with the title "Attack Detection Application with Attack Tree for Mobile System using Log Analysis" [3], the authors developed an Android OS-based detection application for a personal information disclosure attack on smartphones. For attackers' privacy spoofing attacks, applications can compare and analyze attack trees to identify the type of path a mobile attack passes through.

The fourth article titled "Application of Emotion Recognition and Modification for Emotional Telugu Speech Recognition" [4] proposed a method to improve performance on synthetically generated emotional words using the rhythm correction method. Because the majority of Automatic Speech Recognition System (ASR) is trained in a neutral sense, it can affect the performance of the system for emotional speech. ASR technology is important in terms of human-machine interaction.

The fifth article, "Recommending the Best Merchant from Previous Transactions" [5] proposed a system that recommends the best traders from previous transactions. To do this, association rules are extracted based on the distance between merchants, the categories of merchants, 
the number of times items are purchased together, and the user's preference.

The last article titled "Design of Efficient Key Video Frame Protection Scheme for Multimedia Internet of Things (IoT) in Converged 5G Network" [6] propose a transmission method that improves Quality of Service (QoS) to support various high quality multimedia contents in the 5th generation convergence network.

In particular, this paper proposes a priority-based key frame protection method for QoS enhancement in $5 \mathrm{G}$ convergence network.

Acknowledgements The guest editors are thankful to our reviewers for their effort in reviewing the manuscripts. We also thank the Editin-Chief, Dr. Imrich Chlamtac for his supportive guidance during the entire process.

\section{References}

1. Kim H, Yang G, Jung H, Lee SH, Ahn JJ (2018) An intelligent product recommendation model to reflect the recent purchasing patterns of customers, Mobile Netw Appl, pp 1-8. https://doi.org/10.1007/s11036-017-0986-7

2. Ye H, Dong K, Gu T, Huang Z (2018) SMinder: Detect a leftbehind phone using sensor-based context awareness, Mobile Netw Appl, pp 1-13. https://doi.org/10.1007/s11036-017-0987-6

3. Kim D, Shin D, Shin D, Kim YH Attack detection application with attack tree for mobile system using log analysis, Mobile Netw Appl, pp 1-9. https://doi.org/10.1007/s11036-018-1012-4

4. Vegesna VVR, Gurugubelli K, Vuppala AK (2018) Application of Emotion Recognition and Modification for Emo- tional Telugu Speech Recognition, Mobile Netw Appl, pp 1-9. https://doi.org/10.1007/s11036-018-1052-9

5. Chatterji S (2018) Recommending the best merchant from previous transactions, Mobile Netw Appl, pp 1-6. https://doi.org/10.1007/s11036-018-1053-8

6. Lee JH, Hong GS, Lee YW, Kim CK, Park N, Kim BG (2018) Design of efficient key video frame protection scheme for multimedia internet of things (IoT) in converged 5G network, Mobile Netw Appl, pp 1-13. https://doi.org/10.1007/s11036-018-1107-y

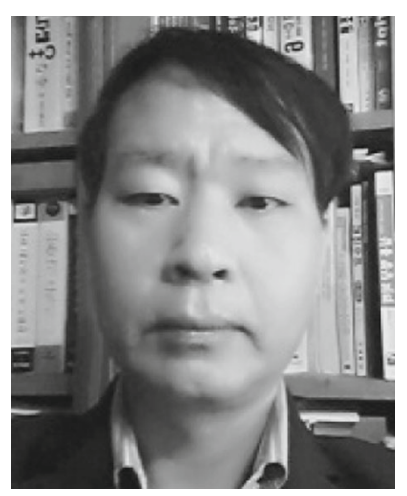

Cheonshik Kim is currently a professor at the Sejong University, Seoul, and Republic of Korea (South). He authored more than 100 publications and solely authored four books. His research interests are in security and privacy, data mining, data hiding, watermarking, and pattern recognition. Prof. Kim serves as an organization and program committee member for numerous international conferences and workshops. He served or is serving as an Editor or/and Guest Editor for many technical journals, such as Real-Time Image Processing (Springer), Transaction on Advanced Communications Technology (TACT), Personal and Ubiquitous Computing (Springer), Journal of Computer Networks and Communications (Hindawi), Sensors (MDPI), Symmetry (MDPI). He is Vice-President since 2010 as IEEK Computer Society. He is listed in Who's Who in the World 2013-2014 (30-31st Edition), 2017, and 2018. He is a member of the IEEE. 


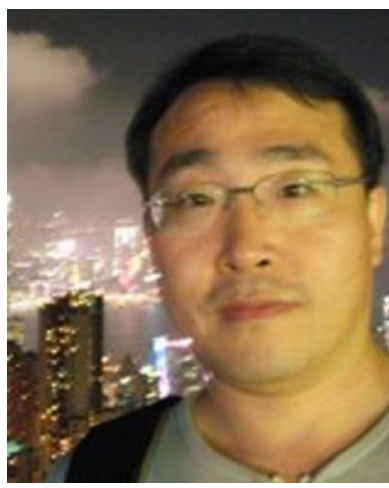

Byung-Gyu Kim has received his BS degree from Pusan National University, Korea, in 1996 and an MS degree from Korea Advanced Institute of Science and Technology (KAIST) in 1998. In 2004, he received a $\mathrm{PhD}$ degree in the Department of Electrical Engineering and Computer Science from Korea Advanced Institute of Science and Technology (KAIST). In March 2004, he joined in the real-time multimedia research team at the Electronics and Telecommunications Research Institute (ETRI), Korea where he was a senior researcher. In ETRI, he developed so many real-time video signal processing algorithms and patents and received the Best Paper Award in 2007.From February 2009 to February 2016, he was associate professor in the Division of Computer Science and Engineering at SunMoon University, Korea. In March 2016, he joined the Department of Information Technology (IT) Engineering at Sookmyung Women's University, Korea where he is currently an associate professor. In 2007, he served as an editorial board member of the International Journal of Soft Computing, Recent Patents on Signal Processing, Research Journal of Information Technology, Journal of Convergence Information Technology, and Journal of Engineering and Applied Sciences. Also, he is serving as an associate editor of Circuits, Systems and Signal Processing (Springer), The Journal of Supercomputing (Springer), The Journal of Real-Time Image Processing (Springer), and International Journal of Image Processing and Visual Communication (IJIPVC). From March 2018, he is serving as the Editor-in-Chief of The Journal of Multimedia Information System and an associate editor of IEEE Access Journal. He has also served or serves as Organizing Committee of CSIP 2011, a Co-organizer of CICCAT2016/2017, and Program Committee Members of many international conferences. He has received the Special Merit Award for Outstanding Paper from the IEEE Consumer Electronics Society, at IEEE ICCE 2012, Certification Appreciation Award from the SPIE Optical Engineering in 2013, and the Best Academic Award from the CIS in 2014. He has been honored as an IEEE Senior member in 2015. He is serving as a professional reviewer in many academic journals including IEEE, ACM, Elsevier, Springer, Oxford, SPIE, IET, MDPI, and so on. He has published over 200 international journal and conference papers, patents in his field. His research interests include image and video signal processing for the content-based image coding, video coding techniques, 3D video signal processing, deep/reinforcement learning algorithm, embedded multimedia system, and intelligent information system for image signal processing. He is a senior member of IEEE and a professional member of ACM, and IEICE.

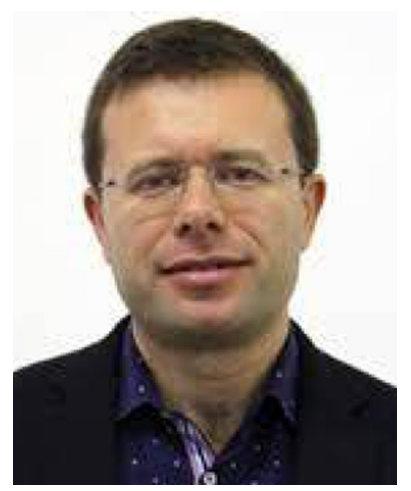

Joel J. P. C. Rodrigues is a professor at the National Institute of Telecommunications (Inatel), Brazil and senior researcher at the Instituto de Telecomunicações, Portugal. Prof. Rodrigues is the leader of the Internet of Things research group (CNPq), Director for Conference Development - IEEE ComSoc Board of Governors, IEEE Distinguished Lecturer, Technical Activities Committee Chair of the IEEE ComSoc Latin America Region Board, the President of the scientific council at ParkUrbis - Covilhã Science and Technology Park, the Past-Chair of the IEEE ComSoc Technical Committee on eHealth, the Past-chair of the IEEE ComSoc Technical Committee on Communications Software, Steering Committee member of the IEEE Life Sciences Technical Community and Publications co-Chair, and Member Representative of the IEEE Communications Society on the IEEE Biometrics Council. He is the editor-in-chief of the International Journal on E-Health and Medical Communications, the editor-in-chief of the Journal of Multimedia Information Systems, and editorial board member of several high-reputed journals. He has been general chair and TPC Chair of many international conferences, including IEEE ICC, IEEE GLOBECOM, IEEE HEALTHCOM, and IEEE LatinCom. He has authored or coauthored over 650 papers in refereed international journals and conferences, 3 books, and 2 patents. He had been awarded several Outstanding Leadership and Outstanding Service Awards by IEEE Communications Society and several best papers awards. Prof. Rodrigues is a licensed professional engineer (as senior member), member of the Internet Society, and a senior member ACM and IEEE. 